\title{
A Novel Role for Kv1.3 Blockers: Protecting Neural Progenitor Cells from a Hostile Inflammatory Environment
}

\author{
Haiyan Peng ${ }^{1,2 *}$ and David J. Huss ${ }^{3,4,5 *}$ \\ ${ }^{1}$ Department of Neurology, ${ }^{2}$ Neuroscience Graduate Studies Program, ${ }^{3}$ Department of Molecular Virology, Immunology, and Medical Genetics, ${ }^{4}$ Molecular, \\ Cellular, and Developmental Biology Program, and ${ }^{5}$ Center for Clinical and Translational Science, The Ohio State University, Columbus, Ohio 43210 \\ Review of Wang et al.
}

Multiple sclerosis (MS) is a debilitating neurological disorder that affects $\sim 2.5$ million individuals worldwide. While the precise etiology of the disease is unknown, both immune-mediated inflammation and neurodegeneration play a role in disease progression. Myelin-specific activated T-cells infiltrate the CNS and perpetuate inflammation that causes damage to the myelin sheath of axons, ultimately leading to axonal transection and loss. As the disease progresses, there is increased gray matter involvement and brain atrophy, despite a reduction in inflammation. Currently, there are six Food and Drug Administration-approved immunomodulatory therapies that reduce new inflammatory lesions and clinical exacerbations; however, these therapies become less effective as the disease progresses. Thus, there is a need for novel neuroprotective and neurorestorative therapies for individuals with MS.

Neural progenitor cells (NPCs) are multipotent cells found in the adult CNS with the ability to migrate, proliferate, and differentiate into both neurons and glial cells. Based on these properties, there is much interest in using NPCs for thera-

\footnotetext{
Received June 1, 2010; revised June 29, 2010; accepted July 1, 2010. We thank John Gensel for insightful editing.

*H.P. and D.J.H. contributed equally to this work.

Correspondence should be addressed to David J. Huss or Haiyan Peng,

The Ohio State University, 460 West 12th Avenue, Columbus, $\mathrm{OH} 43210$,

E-mail: Huss.25@osu.edu or Peng.87@0su.edu.

D0I:10.1523/JNEUROSCI.2802-10.2010

Copyright $(\underset{2}{ } 2010$ the authors $\quad 0270-6474 / 10 / 3010609-03 \$ 15.00 / 0$
}

peutic purposes in neurological diseases such as MS. Although endogenous NPCs can promote functional recovery early in disease through remyelination, they have proven to be of limited benefit in chronic CNS inflammation due to impaired neurogenesis (Pluchino et al., 2008). However, exogenous transplantation of NPCs has shown benefit in a murine model for MS, experimental autoimmune encephalomyelitis (EAE), most likely through mechanisms other than simple cell replacement (Pluchino et al., 2005). NPCs reduce inflammation and promote remyelination by releasing neurotrophic factors (brain-derived neurotrophic factor, glial cell line-derived neurotrophic factor, and ciliary neurotrophic factor) and immunoregulatory cytokines (transforming growth factor- $\beta$ ) at lesion sites. Despite these benefits, little evidence has been found that NPCs differentiate into neuronal cells and aid in tissue regeneration in vivo. One possible barrier is that the inflammatory CNS microenvironment established by myelin-specific CD4+ and CD8 + T-cells inhibits NPC neurogenesis. Thus, to successfully implement NPCs as a therapeutic option, researchers must induce NPCs to overcome the unfavorable microenvironment and drive tissue regeneration.

A recently published study by Wang et al. (2010) examined the interaction between NPCs and activated CD8 + T-cells. Using human NPCs and purified CD8+ T-cells, the authors began by identifying the effects of factors released from activated CD8 + T-cells on NPC proliferation and neurogenesis [Wang et al. (2010), their Fig. 1]. They demonstrated that supernatants from activated CD8 + T-cells inhibited proliferation and neuronal differentiation of NPCs. Because granzyme B $(\mathrm{GrB})$ is a major factor released by cytotoxic T-cells and is able to cause oligodendrocyte apoptosis (Saxena et al., 2008), the authors hypothesized that this molecule acted on NPCs. Consistent with this hypothesis, neutralizing GrB in the supernatant restored proliferation and neuronal differentiation. In contrast, adding recombinant GrB to NPC cultures reduced proliferation and neuronal differentiation, suggesting that $\mathrm{GrB}$ is the primary molecule released by activated CD8 + T-cells that suppresses proliferation and differentiation of NPCs.

Previously, Wang et al. (2006) reported that GrB signals neuronal cells through a pertussis toxin (PTX)-sensitive Gi $\alpha$-coupled membrane receptor. In the present study, they replicated this finding in NPCs by pretreating NPCs with PTX before adding GrB [Wang et al. (2010), their Fig. 2]. PTX pretreatment partially blocked the Gi $\alpha /$ Go receptor and thus restored NPC proliferation. Additionally, treatment with $\mathrm{GrB}$ reduced intracellular levels of cAMP. Because cAMP activates the transcription factor CAMP response element-binding protein (CREB), which triggers transcription of genes that pro- 
mote neural cell proliferation (Dworkin et al., 2007), it is plausible that activated CD8 + T-cells reduce NPC proliferation via GrB-mediated downregulation of cAMP.

Another important finding by Wang et al. (2010) is that GrB caused increased expression of the voltage-gated potassium channel Kv1.3 in NPCs [Wang et al. (2010), their Fig. 3]. This discovery is significant, because Liebau et al. (2006) demonstrated that pharmacological inhibition of Kv1.3 increased NPC proliferation. To determine whether upregulation of Kv1.3 was involved in GrB-mediated inhibition of NPC neurogenesis, Wang et al. pretreated NPCs with margatoxin, a Kv1.3 blocker [Wang et al. (2010), their Fig. 4]. Margatoxin reversed GrB-mediated inhibition of NPC neurogenesis, suggesting the importance of the Kv1.3 channel in this effect. Furthermore suppressing Kv1.3 expression with specific small interfering RNAs restored NPC neurogenesis. Finally, the authors examined whether the same effects were observed in vivo in a rat model. $\mathrm{GrB}$ or $\mathrm{GrB}+$ margatoxin were injected into rat dentate gyrus [Wang et al. (2010), their Fig. 5]. After $7 \mathrm{~d}$, rats were injected with BrdU and killed $2 \mathrm{~h}$ later for analysis. Consistent with the in vitro proliferation data, GrB injection reduced BrdU-labeled cells, but coinjection of margatoxin with GrB restored proliferation.

To demonstrate the clinical relevance of this work, Wang et al. (2010) measured GrB levels in the CSF of patients with MS [Wang et al. (2010), their Fig. 7] and found that these patients had higher GrB levels than control subjects. Although these patients were identified as having clinically defined relapsing-remitting MS, it was not stated whether the patients were in relapse or remission. A more comprehensive study was recently performed that demonstrated GrB levels are increased in the CSF of MS patients during relapse, but not during remission (Malmeström et al., 2008). Interestingly, the increases seen during relapse were confined to the CNS compartment and not observed in patient sera. However, in both of these studies, it is unclear whether the increases in $\mathrm{GrB}$ levels in the CSF are causal or merely a byproduct of the inflammation.

One possible explanation for the differential expression of $\mathrm{GrB}$ during relapse and remission is that transient release of GrB during CD8 + T-cell infiltration contributes to oligodendrocyte damage and the subsequent clinical relapse, whereas resolution of the inflammation allows remyelination and promotes remission. Al-
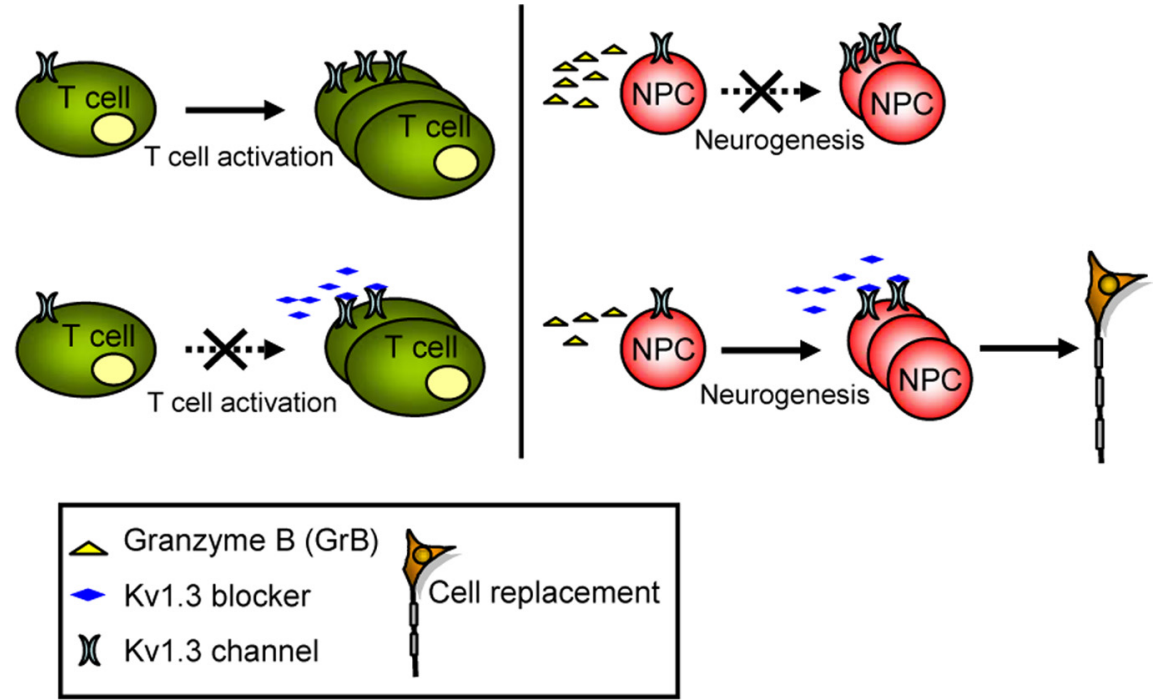

Figure 1. Dual role of Kv1.3 blocker. Kv1.3 expression increases in T-cells upon activation (upper left), and blocking its expression inhibits T-cell activation and proliferation (lower left). Wang et al. (2010) demonstrated that inflammation also increases Kv1.3 levels on NPCs, which results in impaired neurogenesis (upper right), and that Kv1.3 blockers reverse this effect (lower right) and promote differentiation.

though this may be significant, most other inflammatory markers (IFN- $\gamma$, TNF- $\alpha$, IL-17, etc.) are increased in the CSF of MS patients during relapse, while immunomodulating factors (TGF- $\beta$, IL-10, etc.) are associated with remission. The extent to which this is clinically relevant to diagnosis and treatment remains to be determined.

The experiments performed by Wang et al. (2010) establish that the pro-inflammatory molecule $\mathrm{GrB}$, released from activated CD8 + T-cells, prevents NPC proliferation and neurogenesis. In MS, the inflammatory lesion is very complex, with numerous immune cell types playing critical roles. In addition to CD8 + T-cells, $\mathrm{CD} 4+\mathrm{T}$-cells also play a critical role in disease progression. This raises the question of how inflammatory factors such as IFN- $\gamma$, TNF- $\alpha$, and IL-17, released from activated CD4+ T-cells, affect proliferation and neurogenesis of NPCs. To date, there are conflicting reports regarding the effects of pro-inflammatory and antiinflammatory cytokines on neurogenesis. Systematically reproducing experiments like Wang et al. (2010) with both in vitro and in vivo data would provide valuable comparative results for delineating the role of individual cytokines on NPC function.

Finally, it is of interest to note that Kv1.3 is highly expressed on activated CD4+ and CD8+ T-cells that infiltrate the CNS in patients with MS and is important for T-cell proliferation (Rus et al., 2005). In experiments using the EAE model, Kv1.3 blockers reduced T-cell proliferation and activation, prevented the onset of disease, and treated active disease (Beeton et al., 2001). The findings of Wang et al. (2010) further highlight the potential benefit of Kv1.3 blockers, which may serve as a two-edged approach to treating MS by reducing T-cell proliferation and activation and increasing NPC proliferation and neuronal differentiation (Fig. 1).

\section{References}

Beeton C, Wulff H, Barbaria J, Clot-Faybesse O, Pennington M, Bernard D, Cahalan MD, Chandy KG, Béraud E (2001) Selective blockade of $\mathrm{T}$ lymphocyte $\mathrm{K}(+)$ channels ameliorates experimental autoimmune encephalomyelitis, a model for multiple sclerosis. Proc Natl Acad Sci USA 98: 13942-13947.

Dworkin S, Heath JK, deJong-Curtain TA, Hogan BM, Lieschke GJ, Malaterre J, Ramsay RG, Mantamadiotis T (2007) CREB activity modulates neural cell proliferation, midbrainhindbrain organization and patterning in zebrafish. Dev Biol 307:127-141.

Liebau S, Pröpper C, Böckers T, Lehmann-Horn F, Storch A, Grissmer S, Wittekindt $\mathrm{OH}$ (2006) Selective blockage of Kv1.3 and Kv3.1 channels increases neural progenitor cell proliferation. J Neurochem 99:426-437.

Malmeström C, Lycke J, Haghighi S, Andersen O, Carlsson L, Wadenvik H, Olsson B (2008) Relapses in multiple sclerosis are associated with increased CD8 + T-cell mediated cytotoxicity in CSF. J Neuroimmunol 196:159-165.

Pluchino S, Zanotti L, Rossi B, Brambilla E, Ottoboni L, Salani G, Martinello M, Cattalini A, Bergami A, Furlan R, Comi G, Constantin G, Martino G (2005) Neurosphere-derived mul- 
tipotent precursors promote neuroprotection by an immunomodulatory mechanism. Nature 436:266-271.

Pluchino S, Muzio L, Imitola J, Deleidi M, AlfaroCervello C, Salani G, Porcheri C, Brambilla E, Cavasinni F, Bergamaschi A, Garcia-Verdugo JM, Comi G, Khoury SJ, Martino G (2008) Persistent inflammation alters the function of the endogenous brain stem cell compartment. Brain 131:2564-2578.

Rus H, Pardo CA, Hu L, Darrah E, Cudrici C, Niculescu T, Niculescu F, Mullen KM, Allie
R, Guo L, Wulff H, Beeton C, Judge SI, Kerr DA, Knaus HG, Chandy KG, Calabresi PA (2005) The voltage-gated potassium channel Kv1.3 is highly expressed on inflammatory infiltrates in multiple sclerosis brain. Proc Natl Acad Sci USA 102:1109411099.

Saxena A, Bauer J, Scheikl T, Zappulla J, Audebert M, Desbois S, Waisman A, Lassmann $\mathrm{H}, \mathrm{Li}_{-}$ blau RS, Mars LT (2008) Cutting edge: multiple sclerosis-like lesions induced by effector CD8 T cells recognizing a sequestered antigen on oligodendrocytes. J Immunol 181:16171621.

Wang T, Allie R, Conant K, Haughey N, TurchanChelowo J, Hahn K, Rosen A, Steiner J, Keswani S, Jones M, Calabresi PA, Nath A (2006) Granzyme B mediates neurotoxicity through a G-protein-coupled receptor. FASEB J 20:1209-1211.

Wang T, Lee MH, Johnson T, Allie R, Hu L, Calabresi PA, Nath A (2010) Activated T-cells inhibit neurogenesis by releasing granzyme B: rescue by Kv1.3 blockers. J Neurosci 30:5020-5027. 